\title{
Pre-clinical QT Risk Assessment in Pharmaceutical Companies - Issues of Current QT Risk Assessment -
}

\author{
Kiyoshi TaKasuna*, Chiba Katsuyoshi, and Sunao Manabe \\ Medicinal Safety Research Laboratories, Daiichi Sankyo Co., Ltd., Tokyo, Japan
}

(Received January 8, 2009, Accepted January 20, 2009)

\begin{abstract}
Since the Committee for Proprietary Medicinal Products (CPMP) of the European Union issued in 1997 a "points to consider" document for the assessment of the potential for QT interval prolongation by non-cardiovascular agents to predict drug-induced torsades de pointes (TdP), the QT liability has become the critical safety issue in the development of pharmaceuticals. As TdP is usually linked to delayed cardiac repolarization, international guideline (ICH S7B) has advocated the standard repolarization assays such as in vitro $\mathrm{I}_{\mathrm{kr}}$ (hERG current) and in vivo QT interval, or in vitro APD (as a follow up) as the best biomarkers for predicting the TdP risk. However, the recent increasing evidence suggests that the currently used above biomarkers and/or assays are not fully predictive for TdP, but also does not address potential new druginduced TdP due to the selective disruption of $h E R G$ protein trafficking to the cell membrane or VT and/or VF with QT shortening. There is, therefore, an urgent need for other surrogate markers or assays that can predict the proarrhythmic potential of drug candidate. In this review, we provide an ideal pre-clinical strategy to predict the potentials of QT liability and lethal arrhythmia of the drug candidates with recent issues in this field in mind, not at the expense of discarding therapeutically innovative drugs.
\end{abstract}

Keywords: QT risk, QT liability, hERG, Pre-clinical strategy

\section{INTRODUCTION}

In the first half of the 1990s, not only anti-arrhythmic drugs but also non-cardiovascular drugs such as astemizole and terfenadine (anti-histamines), cisapride (prokinetics) and pimozide (antipsychotic) were found to cause QT interval (the time elapsed between ventricular depolarization and repolarization) prolongation and polymorphic ventricular tachyarrhythmia, known as torsades de pointes (TdP). In response to these reports, the Committee for Proprietary Medicinal Products (CPMP) of the European Union issued in 1997 a "points to consider" document for the assessment of the potential for QT interval prolongation by non-cardiovascular agents. The impact of the document on drug discovery and development has been significant, and the QT liability has become the number one safety issue in the development of pharmaceuticals, su-

${ }^{*}$ Corresponding author

Tel: +81-3-5696-9080 Fax: +81-3-5696-8335

E-mail: takasuna.kiyoshi.a3@daiichisankyo.co.jp perseding liver injury being the primary cause of drug withdrawals in the last decade. It currently appears that Public Health Authorities expect all new drug candidates to be tested in cardiac electrophysiological studies. Indeed, the CPMP document was followed by the newly adopted International Conference on Harmonization (ICH) S7B (pre-clinical, implemented in Europe in November 2005, in USA in October 2005, but still awaiting adoption in Japan) and E14 (clinical) guidance documents, both of which confirm this requirement.

Drug-induced TdP with non-cardiovascular drugs is a rare (estimated to $1 / 100,000$ or less exposed patients) (Camm, 2005; Vik, 2008) but potentially life threatening arrhythmia leading to syncope and, even more rarely, to ventricular fibrillation (VF) and sudden cardiac death. Therefore, even a low risk is not justified for drugs intended to treat non-life threatening diseases unless it is a major therapeutic breakthrough and no other safer alternative exist. Increasing awareness of the potential for drug-induced arrhythmia has resulted in re-labelling, warnings, and with- 
Table I. Drugs resulted in re-labelling, warnings, and withdrawals due to QT liability

\begin{tabular}{ll}
\hline Withdrawn from market & Prenylamine (anti-anginal) \\
& Terodiline (anti-incontinence) \\
& Astemizole/Terfenadine \\
& (anti-histamine) \\
& Grepafloxacin (anti-biotic) \\
& Cisapride (prokinetic) \\
& Sertindole/Droperidole \\
& (anti-psychotic) \\
& Pimozide/Thioridazine \\
Prescribing restriction & (anti-psychotic) \\
& Halofantrine (anti-malarial) \\
Approval delayed & Ziprasidone (anti-psychotic) \\
Labelling implication & Far too many example \\
\hline
\end{tabular}

drawals of some drugs from the market (Table I).

$\mathrm{TdP}$ is usually linked to delayed cardiac repolarization, as manifested by a prolongation of the QT interval on the surface electrocardiogram (ECG). As a consequence, drug-induced QT prolongation has been generally considered as a surrogate marker for drug-induced TdP. Although not all the drugs that prolong the QT interval in clinical use have been reported to induce TdP, the drug development process should identify as early as possible those compounds having the potential of QT prolongation because of no other ideal biomarkers predicting the proarrhythmic activity with absolute reliability.

In this review, we provide 1) Potential pre-clinical approach for QT risk assessment, 2) Pre-clinical strategy to assess QT liability with recent issues in this field in mind, and 3) Useful animal models for predicting proarrhythmic potential of drug candidates.

\section{POTENTIAL PRE-CLINICAL APPROACH FOR QT RISK ASSESSMENT}

The QT interval is defined as the time interval between the onset of the QRS complex and the end of the T wave (Fig. 1), which corresponds to the duration of ventricular action potential (APD), plus the time associated with transmission across the myocardium. Therefore the prolongation of the APD corresponds to an increase in the QT interval. The activation of the cardiac ventricular action potential (AP) is initiated by a rapid opening of voltage-gated sodium channels ( $I_{\mathrm{Na}}$ : Phase 0 ). It is followed by a transient repolarization in the transmembrane potential, due to the inactivation of $\mathrm{I}_{\mathrm{Na}}$ and the activation of the transient outward potassium current ( $I_{\text {to }}$ ) (Phase 1). Phase 2 "plateau level" is generated by the inward L-type calcium current

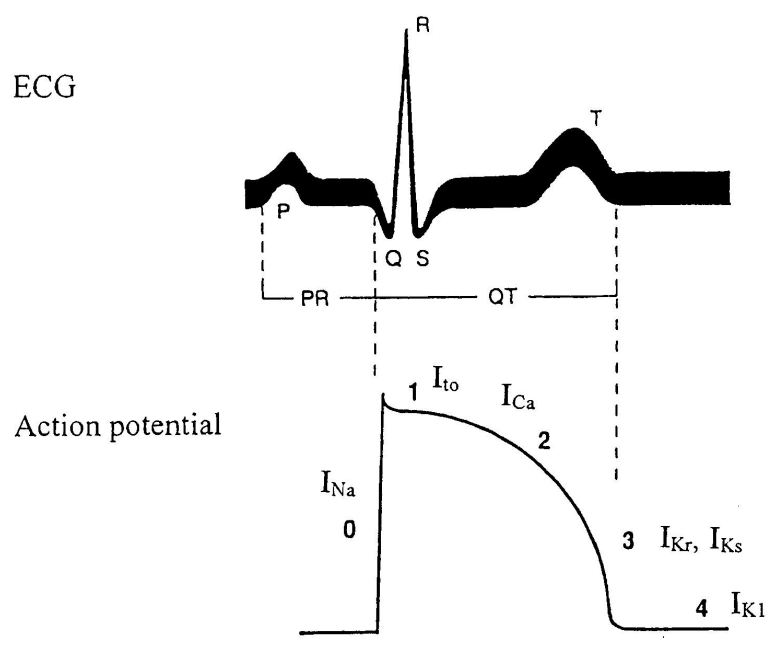

Fig. 1. Ionic molecular basis.

( $\left.\mathrm{I}_{\mathrm{Ca}} \mathrm{L}\right)$ and the balance it reaches with repolarizing potassium currents, and the cell membrane slowly repolarizes as potassium efflux exceeds calcium influx. Phase 3 is the final phase of the repolarization, resulting from the activation of the two components of the delayed rectifier potassium current, $\mathrm{I}_{\mathrm{kr}}\left(\mathrm{r}=\right.$ rapid component) and $\mathrm{I}_{\mathrm{ks}}$ (s=slow component). The repolarization phase correlates with the $T$ wave on the surface ECG. Finally, the inward rectifier potassium current $\mathrm{I}_{\mathrm{k} 1}$ completes the late phase of the repolarization, Phase 4.

QT prolongation can theoretically be caused by a reduction of outward currents ( $\mathrm{I}_{\mathrm{Kr}}$ and $\mathrm{I}_{\mathrm{Ks}}$ ) and/or enhancement of inward currents $\left(I_{\mathrm{Na}}\right.$ and $\left.\mathrm{I}_{\mathrm{Ca}} \mathrm{L}\right)$ during phases 2 and 3 of the AP. Most of QT-prolonging drugs, however, have been shown to reduce the $\mathrm{I}_{\mathrm{Kr}}$ by inhibiting the $\mathrm{K}^{+}$channel encoded by the human ether-a-go-go-related gene (hERG). Therefore, blockade of the hERG $\mathrm{K}^{+}$channel is the most important mechanism through which QT-prolonging drugs prolong the APD.

The CPMP 'points to consider' document and the $\mathrm{ICH}$ S7B guidelines proposed pre-clinical screening methods that is likely to unveil the QT prolongation potential of drug candidates before its' first use in man. Recently, industry sponsored initiatives, such as ILSI/HESI (Hanson et al., 2006), QT PRODACT (Hashimoto, 2008), and a large retrospective analysis (Redfern et al., 2003), designed to generate comparative data using known pro-arrhythmic agents and negative controls, have indicated that pre-clinical screening commonly used is quite robust, with good sensitivity and specificity and with little inter-laboratory differences, but no single test provides enough information upon which to make an adequate prediction of clinical QT liability. On this basis, the ICH S7B adopted the concept of 
developing an "Integrated Risk Assessment".

Essential components of the integrated risk assessment are

lonic current in stable cell lines expressing hERG channel (hERG current): Various heterologous expression systems are available, such as Xenopus laevis oocytes, mouse fibroblasts (C cells), human embryonic kidney (HEK293) cells, and Chinese hamster ovary ( $\mathrm{CHO}$ ) cells, all of which have relatively little endogenous voltage-gated channel activity. Assays commonly conducted use the latter two cell types transfected with the human ether-a-go-go cDNA that encodes the pore-forming $\alpha$ subunits of the channel carrying an easily measurable $\mathrm{I}_{\mathrm{Kr}}$-like current, using whole cell patch clamp technology. There are some technologies to investigate the interaction of drug candidates with $\mathrm{hERG} \mathrm{K}^{+}$channel with high-throughput, such as radioligand binding assays, rubidium flux assays and fluorescence assays using voltage-sensitive dyes. Recently, the automated planar array-based high to middle-throughput voltage clamp systems (e.g., PatchXpress, lonWorks (Quattro) or QPatch) have become available and these, as well as traditional manual patch clamp system, are regarded as "gold standard" screening methods.

In addition, the availability of in silico methods to predict

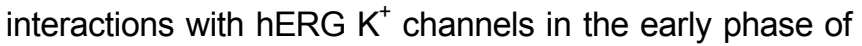
drug development would improve the screening rate and would also lower the costs compared to experimental assay methods.

Action potential duration (APD) measured in isolated cardiac tissues: hERG $\mathrm{K}+$ channel blockade is the key mechanism underlying QT prolongation, but other ionic effects such as increasing the plateau $I_{\mathrm{Na}}, \mathrm{I}_{\mathrm{Ca}} \mathrm{L}$ or effects on other potassium currents (e.g., I I $I_{\mathrm{Ks}} \mathrm{I}_{\mathrm{to}}$, the ultra-rapidly activating delayed rectifier current $I_{K u r}$, and the inward rectifier current $I_{\mathrm{K} 1}$ etc.) may either counterbalance or contribute to this effect. Therefore, multi-cellular preparations, such as isolated dog or rabbit Purkinje fibers or guinea pig papillary muscle or ventricular slice are suitable to assess the effect of drug candidates on multiple ion channels, whose concerted action leads to the overall membrane action potential in cardiac cells. Since APD assays have failed to detect APD prolongation for all known pro-arrhythmic compounds (e.g., terfenadine), this assay was given "follow-up" test status in the ICH S7B guideline. However, due to the recent evidence suggesting the poor correlation between hERG current blocking and APD prolongation, we strongly recommend the APD assay especially at the early exploratory stage.

The QT interval measurements in in vivo model: In vivo models are not only sensitive to detect drug-induced QT prolongation and/or arrhythmia, but also allow for testing in an intact animal system, which is influenced by autonomic tone and for detecting potential effects of unknown active metabolites. Heart rate, blood pressure, and ECG (PR, QRS and QT intervals, T- and U-wave morphology, and arrhythmic episodes) are determined in unrestrained monkeys or dogs (recently guinea pigs, marmosets or minipigs) with implanted telemetry systems. Meanwhile, the ionic mechanisms of repolarization in adult rats and mice differ from larger species including humans, so that use of these species is not considered appropriate. Although there is clear preference for safety pharmacology studies to be performed in conscious free-moving animals in the $\mathrm{ICH}$ S7A guideline, anesthetized (e.g., isoflurane or halothane) animals such as dogs and guinea pigs are generally regarded as more useful because of higher sensitive to drug-induced QT prolongation than conscious animals, and better control of the heart rate (e.g., through cardiac pacing at a constant rate) (Tashibu et al., 2005; Yao et al., 2008).

\section{OVERVIEW OF THE PREDICTIVE VALUES OF THE STANDARD REPOLARIZATION ASSAY - ILSI/HESI AND QT PRODACT ANALYSES -}

The CPMP document brought Pharmaceuticals industry and Public Health Authorities into the discussion of how to best address drug-induced QT prolongation and proarrhythmias. The predictive value of existing tests was unknown at that time. This led to the initiation of industry sponsored prospective, comparative analyses using known pro-arrhythmic agents and negative controls, such as ILSI/HESI and the Japanese QT PRODACT initiative.

Brief summary of ILSI/HESI and QT PRODACT analyses is shown below (Hanson et al., 2006; Hashimoto, 2008) (Table II, III).

ILSI/HESI: Six positive compounds with well established propensity to induce proarrythmias in patients (Bepridil, cisapride, haloperidol, pimozide, terfenadine, and thioridazine) and six negative compounds with well established lack of this effect (Amoxicillin, aspirin, captopril, diphenhydramine, propranolol, and verapamil) were tested. However, compound selection was limited by patent issues and commercial availability of suitable formulations. ILSI/HESI analysis concluded that a combination of hERG and in vivo QT (conscious dog QTcF) assays provided an acceptable prediction of QT prolongation and/or proarrhythmias, and most importantly, with this combination, none of the positive compounds came out as false negatives. 
Table II. Summary of ILSI/HESI comparative analysis

\begin{tabular}{|c|c|c|}
\hline Assay & Test system & Summary \\
\hline \multirow[t]{2}{*}{ In vitro hERG assay } & HEK 293 cell & $\begin{array}{l}\text { Low (less than about 3-fold) inter-laboratory variability in } \mathrm{IC}_{50} \text { between two major, } \\
\text { commercial laboratories (ChanTest and Zenas), with the exception of pimo- } \\
\text { zide (approximately } 40 \text {-fold). }\end{array}$ \\
\hline & & False negative: None False positive: Verapamil \\
\hline \multirow[t]{4}{*}{ In vitro APD assay } & Dog Purkinje fiber & $\begin{array}{l}\text { No significant } A P D\left(\mathrm{APD}_{90}\right) \text { prolongation was detected in } 4 \text { out of } 6 \text { positive com- } \\
\text { pounds }\end{array}$ \\
\hline & & False negative: Pimozide; bepridil; thioridazine; terfenadine \\
\hline & & When triangulation (APD ${ }_{90-40}$ ) was used, a positive signal was detected in 5 out of \\
\hline & & $\begin{array}{l}6 \text { positive compounds. } \\
\text { False negative: Terfenadine False positive: Verapamil }\end{array}$ \\
\hline \multirow[t]{3}{*}{ In vivo QT assay } & Dog with telemetry system & $\begin{array}{l}\text { There was no major gap in the degree of detecting ability of QTc prolongation } \\
\text { among the choice of heart rate correction algorithm (QTcF (Fridericia), QTcB } \\
\text { (Bazett) or QTcIQ (individual), but QTcF consistently identified all positive } \\
\text { compounds. Furthermore, when QTcB was used, } 3 \text { of } 6 \text { negative compounds } \\
\text { showed positive signal. }\end{array}$ \\
\hline & & False negative: None \\
\hline & & False positive: Diphenhydramine; propranolol; verapamil (Hanson et al., 2006) \\
\hline
\end{tabular}

Table III. Summary of QT PRODACT comparative analysis

\begin{tabular}{|c|c|c|}
\hline Assay & Test system & Summary \\
\hline In vitro APD assay & Guinea pig papillary muscle & $\begin{array}{l}\text { No significant positive signal was detected in } 4 \text { out of } 11 \text { positive compounds. } \\
\text { False negative: Astemizole; bepridil; pimozide; terfenadine } \\
\text { When triangulation (APD90-30) was used, positive signal was detected in } 10 \\
\text { out of } 11 \text { positive compounds. } \\
\text { False negative: Terfenadine }\end{array}$ \\
\hline In vivo $\mathrm{QT}$ assay & Dog anesthetized with isoflurane & $\begin{array}{l}\text { When QTcF was used at the correction factor, significant QT prolongation } \\
(>10 \%) \text { was detected in all the positive compounds }(11 / 11) \\
\text { No false negative. No false positive was detected on the negative } \\
\text { compounds. }\end{array}$ \\
\hline In vivo $\mathrm{QT}$ assay & Dog with telemetry system & 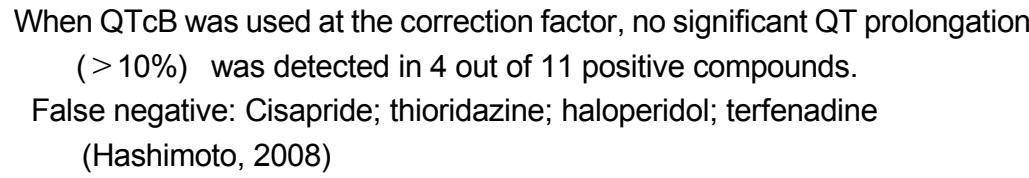 \\
\hline
\end{tabular}

QT PRODACT: Eleven positive compounds with well established propensity to induce proarrythmias in patients [Bepridil, cisapride, haloperidol, pimozide, terfenadine, thioridazine, astemizole, E-4031, MK-499, qunidine, and dl-sotalol (as standard)] and eight negative compounds with well established lack of this effect (Amoxicillin, aspirin, captopril, diphenhydramine, propranolol, verapamil, ciprofloxacin, and nifedipine) were tested. Disopyramide (as positive compound), flecainide, and lidocaine (as negative compounds) were used only for APD assay. QT PRODACT analysis concluded that in vivo QT (anesthetized dog QTcF/conscious monkey QTcB) and APD assay (using triangulation as an index) can provide an acceptable prediction for QT prolongation and/or proarrhythmias. The dogs anesthetized with isoflurane would be more sensitive model for detecting the drug-induced QT prolongation than a conscious model.

\section{PROPOSED PRE-CLINICAL STRATEGY TO ASSESS QT LIABILITY}

The current standard strategy that is to be followed during an early exploratory stage is outlined in Fig. 2 (heavy line course). It is important to note that no single test has a 


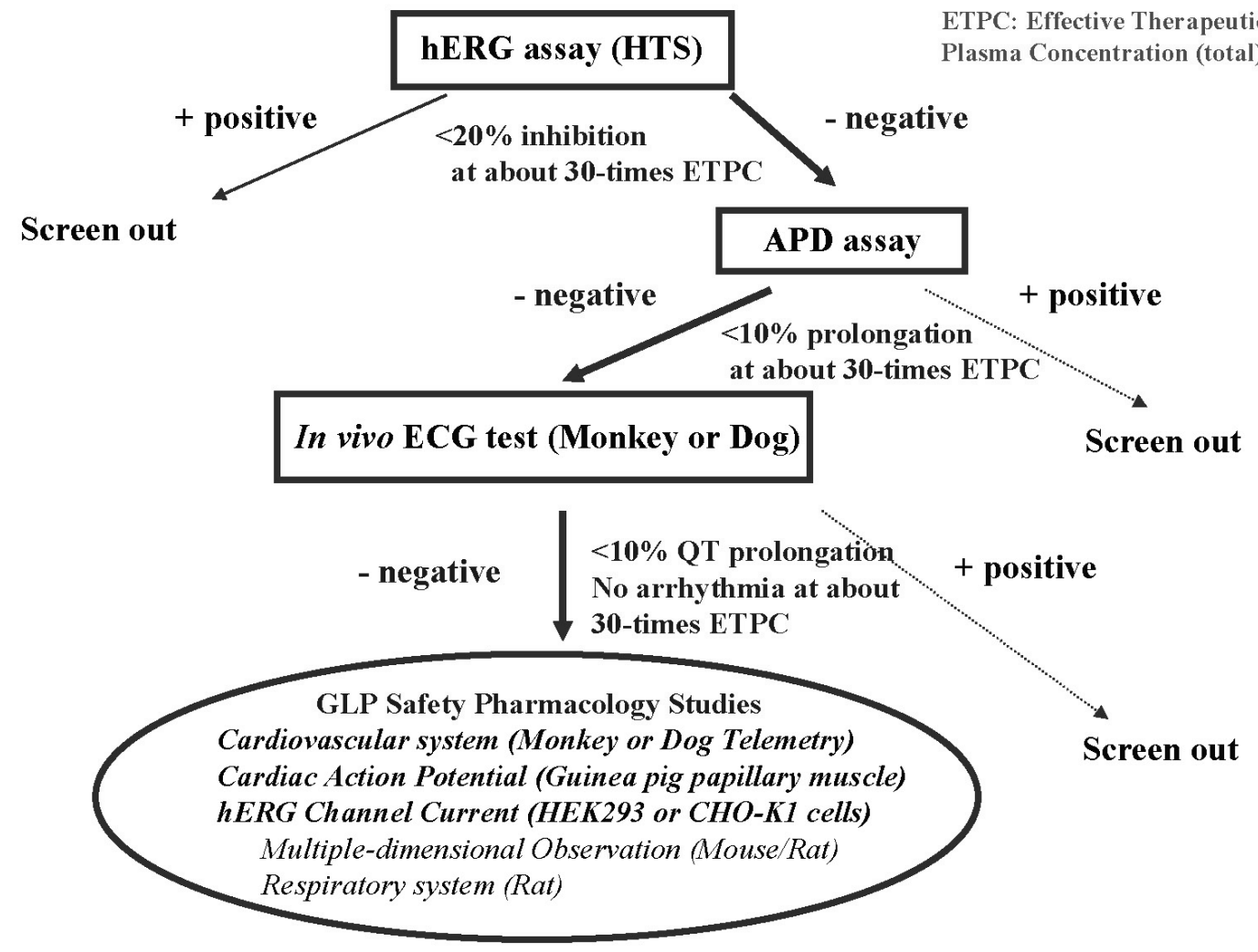

Fig. 2. Proposed strategy for the QT risk assessment of a drug candidate. predictive value with absolute reliability and that an integrated approach with insight into the advantages and limitations of each test can provide a more accurate prediction, which allows decision-making during development. The strategy will need to be revised in line with emergent scientific knowledge in this field. A consistent strategy should be used for all compounds, but the interpretation of its outcome will depend on the extent that a new drug meets unmet medical needs. In the case drug candidate is expected to be a major therapeutic breakthrough and no other safer drug is available to replace it, then these principles will be taken into account when interpreting the positive outcome of the non-clinical QT testing.

Since $\mathrm{hERG} \mathrm{K}^{+}$channel blockade is the main mechanism leading to drug-induced QT prolongation, investigation of the effect on hERG $\mathrm{K}^{+}$current using hERGtransfected cells by middle to high-throughput voltage clamp system can be considered as the primary test. Then, APD assay using guinea pig papillary muscle is encouraged because the hERG assay does not provide answers on all ion channel currents involved in cardiac repolarization. Both these in vitro assays are very useful approach particularly at the early exploratory stages, at which time in vivo studies are not yet feasible due to a limited compound availability.
When a drug candidate has no positive signal in the hERG assay (HTS), and is considered to have potential for further development based on other pre-clinical studies for physicochemical, toxicological and metabolic properties, then in vitro APD assay using guinea pig papillary muscle and in vivo electrophysiological study will be performed using unrestrained monkeys (or dogs) with implanted telemetry systems.

It is likely that almost all compounds would show positive signal in a hERG, APD or in vivo QT assay to some extent. Thus, the ultimate risk assessment should not be based on the presence or absence of effects, but rather on the safety margin between the anticipated therapeutically relevant plasma concentration and the undesired side effect. In each assay, the following criteria for decision-making on progression into further development is fundamentally adopted.

hERG assay: The rational to use of $\mathrm{IC}_{50}$ values is obvious for calculation of the safety margin because $I_{50}$ is the accurate part of the inhibitory concentration response curve and is available for comparison between compounds becomes more robust. $\mathrm{IC}_{50}$, however, is not necessarily determined accurately in the primary hERG assay using HTS at the early exploratory stage because of limited number of cells and evaluated concentrations, and of poor sol- 
ubility etc. Thus, $20 \%$ inhibition $\left(\mathrm{IC}_{20}\right)$ is adopted to be a minimum physiologically significant rough standard instead of calculating $\mathrm{IC}_{50}$. Indeed, some researchers have suggested that $\mathrm{IC}_{10-20}$ levels in $\mathrm{hERG}$ assay appear to more accurately reflect the potential to cause clinically significant QT prolongation and TdP. A literature survey (Cavero et al., 2000; Webster et al., 2002; Redfern et al. 2003) suggests that a minimum 30 -fold margin between hERG IC $\mathrm{C}_{50}$ and effective therapeutic plasma concentrations unbound (ETPC unbound) would be adequate to ensure acceptable degree of "safety" from arrhythmogenesis with a low risk of false positive, setting aside some "outliers" such as terfenadine or tacrolimus (Webster et al., 2002). In consideration of accidental overdose, individual variations in plasma protein binding, drug metabolism or function of $\mathrm{hERG} \mathrm{K}^{+}$channel, and possibility of the local concentrations in myocardium exceeding ETPC $_{\text {unbound, the }}$ criteria at hERG assay (HTS) during the early exploratory stage is set as follows. If a compound inhibits hERG current less than $20 \%$ at 30 -fold ETPC $_{\text {total, }}$ and has no concern of primary pharmacological, physicochemical, toxicological and metabolic properties, proceed to further development, namely APD assay. If there is no available data on not only $\mathrm{IC}_{50}$ at $\mathrm{hERG}$ assay but also ETPC $\mathrm{Ctotal}_{\text {, then }}$ $I_{20}$ and the projected effective concentrations in pre-clinical in vitro primary pharmacology assay $\left(\mathrm{EC}_{\text {assay }}\right)$ are used to calculate the safety margin. However, it is encouraged to use $\mathrm{IC}_{50}$ at $\mathrm{hERG}$ assay and ETPC $\mathrm{unbound}_{\text {for }}$ the calculation of the accurate safety margin at the late of lead optimization stage, before the selection of a drug candidate.

The hERG channel protein is synthetized in the lumen of the rough endoplasmic reticulum, processed by the cytosolic chapelons heat shock protein (Hsp) 70 and Hsp90, and taken to the cell surface. Recently, drugs that inhibit chaperon function have been shown to produce QT prolongation by reduced surface expression due to a trafficking defect of $h E R G$. Therefore, not only $h E R G$ channel direct inhibition but also hERG channel trafficking inhibition assays are recommended to be determined for drugs that might have chaperon inhibitory activity.

APD assay: A 10\% increase in APD at $90 \%$ repolarization $\left(A P D_{90}\right)$ is typically the minimum increase that reaches statistical significance in such studies and is also the degree of change considered physiologically significant by the majority of pharmaceutical companies in a recent survey. The criteria at APD assay is set that if a compound prolongs APD less than $10 \%$ at 30 -fold EC $_{\text {assay }}$ or $E$ PC $_{\text {total, }}$, proceed to further development, namely in vivo QTC assay.

In vivo QTc assay: A 10\% increase in QTc assay is typically the minimum increase that reaches statistical significance in such studies and is also the degree of change

Table IV. Correlation among the integrated in vitro and in vivo QT risk assessments on quinolone antibacterial agents

\begin{tabular}{|c|c|c|c|c|c|c|}
\hline & $\begin{array}{l}\text { hERG current } \\
\text { inhibition (\%) } \\
\text { in HEK293 cell }\end{array}$ & $\begin{array}{l}\text { APD90 } \\
\text { prolongation } \\
\text { (\%) in guinea } \\
\text { pig papillary }\end{array}$ & $\begin{array}{c}\text { Cynomolgus } \\
\text { monkey QTc } \\
\text { prolongation (\%) } \\
\text { telemetry }\end{array}$ & $\begin{array}{l}\text { Beagle dog } \\
\text { MAPD } \\
\text { prolongation } \\
\quad \text { (msec) } \\
\text { anesthetized }\end{array}$ & $\begin{array}{c}\text { Rabbit Carlsson } \\
\text { model } \\
\text { TdP incidence } \\
\text { anesthetized }\end{array}$ & $\begin{array}{l}\text { Beagle dog with } \\
\text { chronic } \\
\text { AV block model } \\
\text { TdP incidence } \\
\text { conscious }\end{array}$ \\
\hline & $100 \mu \mathrm{M}$ & $100 \mu \mathrm{M}$ & $\begin{array}{l}60 \mathrm{mg} / \mathrm{kg} / \mathrm{hr} \\
\text { i.v. infusion }\end{array}$ & $\begin{array}{l}3 \mathrm{mg} / \mathrm{kg} / 10 \mathrm{~min} \\
\text { i.v. infusion }\end{array}$ & $\begin{array}{l}60 \mathrm{mg} / \mathrm{kg} / 20 \mathrm{~min} \\
\text { i.v. infusion }\end{array}$ & $\begin{array}{c}100 \mathrm{mg} / \mathrm{kg} \\
\text { p.o. }\end{array}$ \\
\hline Sparfloxacin & $>70.4$ & 41.2 & No data & 40 & $5 / 5(2 / 5)^{a}$ & $\begin{array}{c}4 / 4 \\
4 / 4(\mathrm{VF}) \\
(6.4 \mu \mathrm{g} / \mathrm{ml})\end{array}$ \\
\hline Moxifloxacin & 42.3 & 14.9 & $\begin{array}{c}24.4-27.3 \\
(24-29 \mu \mathrm{g} / \mathrm{ml}) \\
\text { No arrhythmia }\end{array}$ & $16-36$ & $4 / 7(0 / 5)^{a}$ & $\begin{array}{c}3 / 4 \\
2 / 4(\mathrm{VF}) \\
(5.8-10.8 \mu \mathrm{g} / \mathrm{ml})\end{array}$ \\
\hline Gatifloxacin & 18.4 & 10.1 & $\begin{array}{c}26.3 \\
(38 \mu \mathrm{g} / \mathrm{ml}) \\
\text { No arrhythmia }\end{array}$ & 21 & No data $(1 / 5)^{\mathrm{a}}$ & $\begin{array}{c}3 / 5 \\
1 / 5(\mathrm{VF}) \\
(11.3 \mu \mathrm{g} / \mathrm{ml})\end{array}$ \\
\hline Levofloxacin & 1.8 & 1.3 & $\begin{array}{c}3.5-8.0 \\
(37 \mu \mathrm{g} / \mathrm{ml}) \\
\text { No arrhythmia }\end{array}$ & -2 & No data $(0 / 5)^{a}$ & $\begin{array}{c}0 / 4 \\
(60 \mathrm{mg} / \mathrm{kg} \text { p.o. }) \\
(19.7 \mu \mathrm{g} / \mathrm{ml})\end{array}$ \\
\hline
\end{tabular}

QTc: QT corrected by Bazett formula, TdP: polymorphic ventricular tachycardia, VF: ventricular fibrillation, Values in parenthesis: plasma drug concentration (immediately after end of i.v. infusion or 2 hours after oral dosing), ${ }^{\mathrm{a}} \mathrm{TdP}$ incidence under left thoracotomy for MAP measurement (Cited from Furuhama, 2007, partly modification). 
considered physiologically significant by the majority of pharmaceutical companies in a recent survey. The criteria at in vivo QTc assay is set that if a compound prolongs QTc less than $10 \%$ at 30 -fold ETPC $_{\text {total, }}$, proceed to further development, namely pre-clinical GLP Safety Pharmacology Studies. The standard strategy to reduce the risk of drug-induced QT prolongation and proarrhythmia is well-working properly in case of antibacterial quinolone agents (Table IV, cited from Furuhama, 2007). The hERG current assay can correctly predict not only APD and/or QT prolongation but also proarrhythmic potentials of quinolone antibacterials.

One difficulty of employing the current screen approach, however, is that we might possibly discard therapeutically useful drug candidates since contact research organizations and the pharmaceutical industries including us data suggest approximately $60-85 \%$ of compounds tested show positive signal in $\mathrm{hERG}$ assay, but some of which do not prolong APD or QT interval, and has never associated with TdP (Dumotier and Georgieva, 2007; Lu et al., 2008; Pollard et al., 2008). Fig. 3 shows our recent results of hERG assay (HTS) and APD assay (guinea pig papillary muscle) by each project in 2004-2006. A number of compounds (about $60 \%$ of 2,158 compounds per 2 years) showed positive signal in hERG assay but only approx- imately $30 \%$ of 135 compounds with positive hERG signals prolonged APD (interestingly, of which about $7 \%$ shortened APD). The positive ratios in the hERG and APD assays markedly varied among the projects.

Presumably the reason is that most of compounds have inhibitory effects on cardiac multiple ion channels such as the sodium and L-type calcium channels, and the mixed ion channel activity offsets the effects of inhibition of hERG $\mathrm{K}^{+}$current on APD and QT interval as does verapamil $\left(\mathrm{Ca}^{++}\right.$blocker) or amiodarone (Class III antiarrhythmic). The representative examples are shown in Fig. 4. The multi-ion channel blockers including verapamil have an obvious $h E R G K^{+}$current inhibitory effect but also have $I_{C a}$ inhibitory action at the similar dose-range, which resulted in offset of hERG $\mathrm{K}^{+}$current inhibition-induced APD prolongation. According to the standard screen, these compounds are screen out only due to "hERG positive" although these might be not proarrhythmic, suggesting we might discard the promising candidates.

To resolve the issue, we might have to include another new screen approach. For example, even if a compound inhibits hERG $\mathrm{K}^{+}$current $20 \%$ or more at 30 times ETPC $C_{\text {total }}$, we proceed to APD assay to assure whether the hERG inhibition translates the prolong the APD. Then, if the compound shows a negative signal in APD assay, then

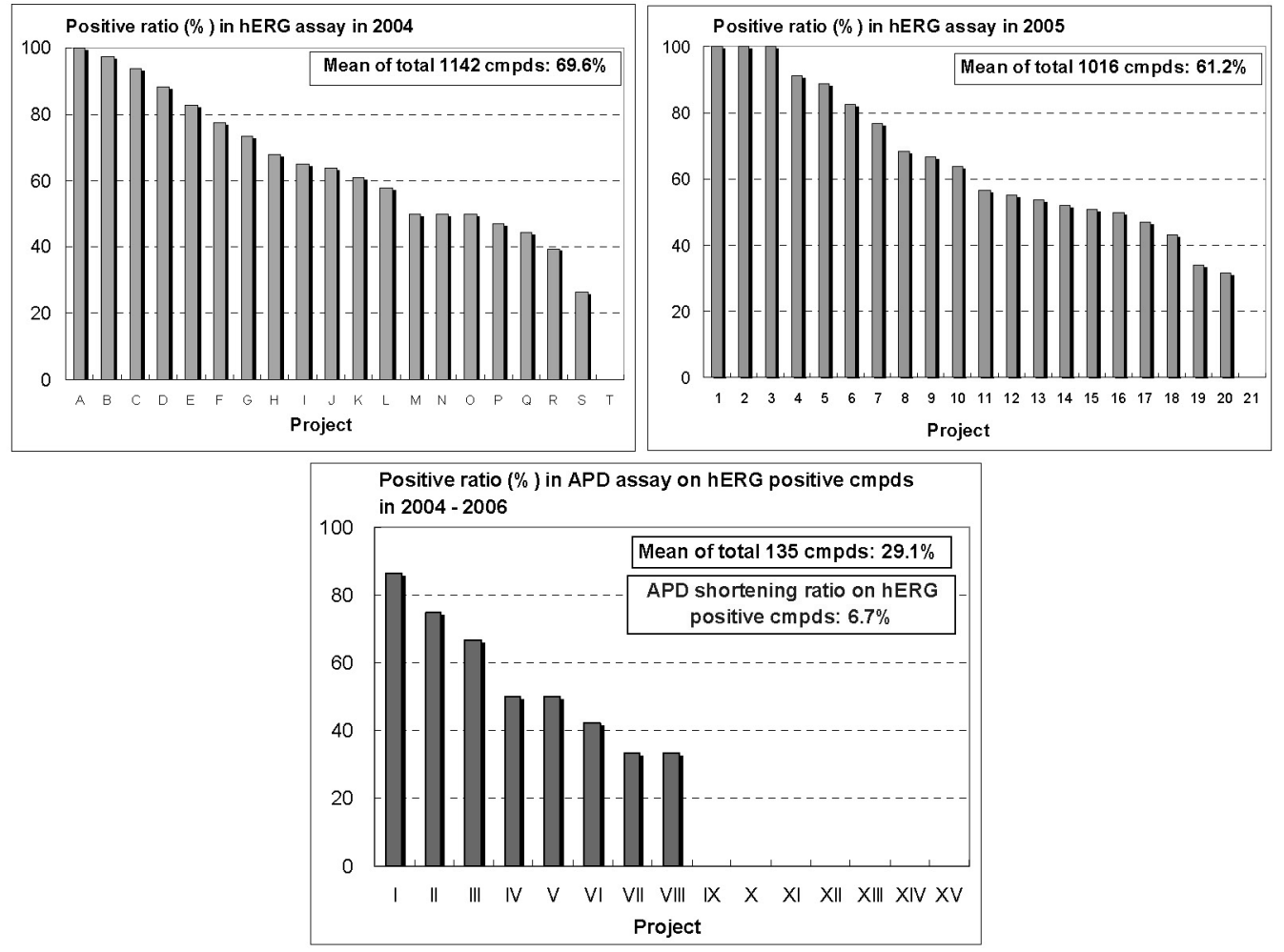

Fig. 3. hERG current $\mathrm{K}^{+}$assay vs. APD assay during 2004-2006. 

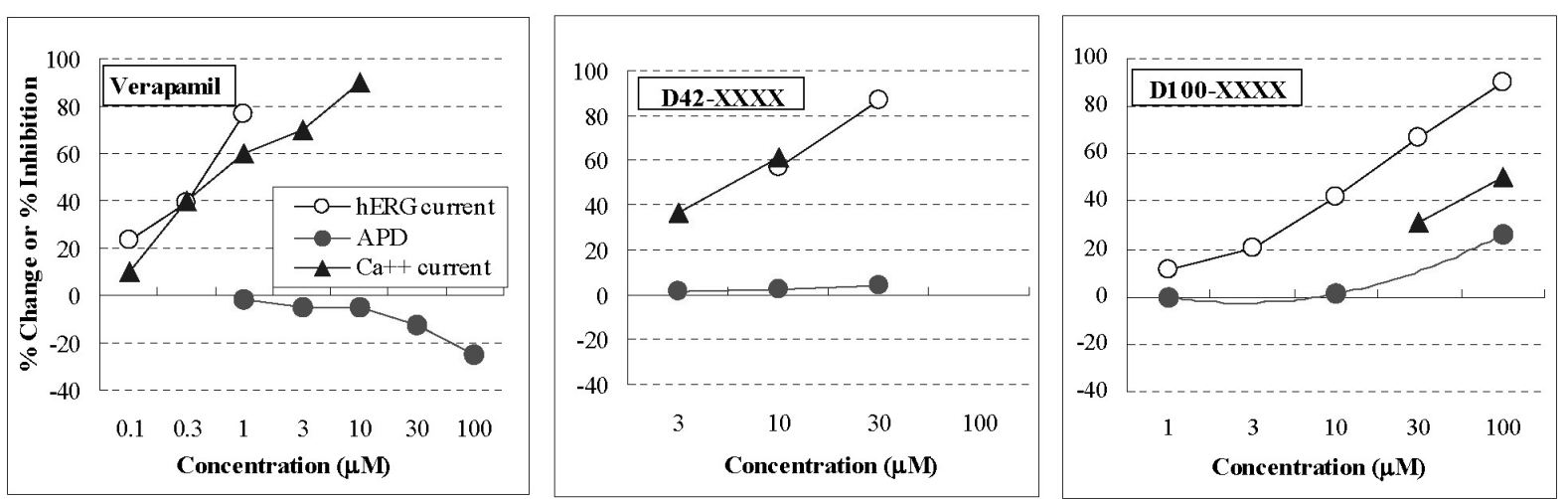

Fig. 4. Effects on APD, hERG $\mathrm{K}^{+}$current and $\mathrm{Ca}^{++}$current of representative multi-channel blockers.

we proceed to QT interval and/or monophasic action potential duration (MAPD) assay in halothane anesthetized dogs or guinea pigs, or Langendorff heart preparation model using rabbits (Screenit system) with pacing heart at a constant cycle length in order to better characterize the QT safety. Furthermore, if we get a few drug candidates which show negative signal in APD or QT/MAPD assay but with positive signal in $\mathrm{hERG}$ assay in this new course, their torsadogenic potential will be assessed using currently available experimental proarrhythmic models as referred to hereinafter to distinguish safer one from less safer drugs. Of course, we must acknowledge that this screen program course is very challenging because there is no consensus for their predictive value of pro-arrhythmic risk, so that this approach are warranted only for drug candidates with a therapeutic potentials greater than existing drugs.

\section{ASSESSING PRO-ARRHYTHMIC RISK OF QT PROLONGING DRUGS}

Although a number of drugs known to cause TdP prolong the APD and/or QT interval by a decrease in the net repolarization current (in most cases by inhibition of the hERG K ${ }^{+}$current), it is now clear that in some cases, neither prolongation of the QT interval nor inhibition of $h E R G$ $\mathrm{K}^{+}$current is sufficient to predict the incidence of TdP (Belardinelli et al., 2005; Dumotier and Georgieva, 2007; Lu et al., 2008). For example, it was shown that azithromycin, a macrolide antibiotic, caused greater APD and QT prolongation than did erythromycin and clarythromycin but did not provoke TdP in million of patients exposed, unlike the latter 2 drugs (Milberg et al., 2002). Furthermore importantly, Valentin et al. (2004) and Lu et al. (2008) suggested that not only QT prolongation but also QT shortening induces potentially more life threatening arrhythmia such as VT and VF, but the current ICH S7B guidelines do not specifically address the possibility of drug-induced fatal arrhythmia associated with QT shortening.

QT interval prolongation per se is no longer a completely reliable surrogate marker to evaluate the torsadogenic potential of a new drug candidate. There is, therefore, an urgent need for a surrogate marker that distinguishes the torsadogenic potential better than the QT interval (Hoffmann and Warner, 2005).The availability of clinically relevant and predictive animal proarrhythmic models implying other surrogate markers that may have higher predictive ability than QT interval or APD prolongation per se have been introduced (Carlsson 2006; Thomsen et al., 2006; Sugiyama 2008). The markers proposed include triangulation, reverse-use dependency, instability (beat to beat variability), spatial dispersion, and terminal repolarization period.

Because drugs with proarrhythmic potentials such as $T d P$, whose incidences are even less than $<1 / 100,000$, can be potentially removed from the market, the experimental model used for predicting the potential of arrhythmia have to be high sensitive and specific to the electrophysiological mechanism of origin of arrhythmia. In addition, preclinical assessment of the risk of drug-induced arrhythmia must recognize that the risk depends foremost on the predisposing factors potentially found in patients exposed to the drug. Namely, incorporation into the experimental protocols of the risk factors that predispose humans to TdP [e.g., female gender, bradycardia, hypokalemia, myocardial ischemia, diabetes, ventricular hypertrophy, prolonged baseline QT interval and genetic variants (polymorphism and mutations)] increases the sensitivity to detect the proarrhythmic potential of drug use in humans. Currently available experimental pro-arrhythmic models that are well validated and established using a large number of drugs (Carlsson 2006; Thomsen et al., 
Table V. Models for assessment of the risk of drug-induced TdP

\begin{tabular}{|c|c|c|c|c|}
\hline Assays & Test systems & Advantages & Disadvantages & References \\
\hline $\begin{array}{l}\text { Langendorff heart preparation } \\
\text { with A-V block } \\
\text { (Screenit system) }\end{array}$ & $\begin{array}{l}\text { Rabbit } \\
\text { Guinea pig }\end{array}$ & $\begin{array}{l}\text { 1. TRlaD can be assessed } \\
\text { simultaneously with MAPD } \\
\text { 2. Rapid data output } \\
\text { 3. Easy to change for factors } \\
\text { related to risk of } \mathrm{TdP}^{\mathrm{b}}\end{array}$ & $\begin{array}{l}\text { 1. Devoid of drug metabolism, } \\
\text { hormonal and nervous systems } \\
\text { 2. Necessity of relatively much } \\
\text { amount of test compound }\end{array}$ & Valentin et al., 2004 \\
\hline $\begin{array}{l}\text { Left ventricular wedge } \\
\text { preparation }\end{array}$ & $\begin{array}{l}\text { Dog } \\
\text { Rabbit } \\
\text { Feline }\end{array}$ & $\begin{array}{l}\text { 1. TRlaD }{ }^{a} \text { can be assessed } \\
\text { simultaneously with MAPD } \\
\text { 2. Easy to change for factors } \\
\text { related to risk of } \mathrm{TdP}^{\mathrm{b}}\end{array}$ & $\begin{array}{l}\text { 1. Devoid of drug metabolism, } \\
\text { hormonal and nervous systems } \\
\text { 2. Necessity of high skillful } \\
\text { 3. Expensive in case of dogs }\end{array}$ & Liu et al., 2006 \\
\hline $\begin{array}{l}\text { Methoxamine-sensitized } \\
\text { anesthetized model } \\
\text { (Carlsson model) }\end{array}$ & Rabbit & $\begin{array}{l}\text { 1. Easy to prepare the model } \\
\text { 2. Relatively sensitive to } \\
\text { directly detect the TdP } \\
\text { potential }\end{array}$ & $\begin{array}{l}\text { 1. Less sensitivity when assessing } \\
\text { drugs with } \alpha \text {-adrenoceptor } \\
\text { blocking properties } \\
\text { 2. Relatively difficult to assess } \\
\text { accurate QT interval }\end{array}$ & Carlsson, 2008 \\
\hline $\begin{array}{l}\text { Chronic A-V block } \\
\text { anesthetized or conscious } \\
\text { model }\end{array}$ & $\begin{array}{l}\text { Dog } \\
\text { Monkey }\end{array}$ & $\begin{array}{l}\text { 1. High sensitive to directly } \\
\text { detect the TdP potential } \\
\text { 2. TRlaD }{ }^{a} \text { can be assessed } \\
\text { simultaneously with MAPD } \\
\text { and easy to change for factor } \\
\text { related to risk of TdP (heart } \\
\text { rate) in anesthetized state } \\
\text { 3. Evaluation by repeated } \\
\text { dosing in conscious state }\end{array}$ & $\begin{array}{l}\text { 1. Necessity of high skillful } \\
\text { 2. Expensive } \\
\text { 3. Large lead time for ventricular } \\
\text { remodeling process ( }>4 \text { weeks } \\
\text { in dogs and }>12 \text { weeks in monke }\end{array}$ & $\begin{array}{l}\text { Oros et al., } 2008 \\
\text { Sugiyama, } 2008\end{array}$ \\
\hline
\end{tabular}

${ }^{a}$ Triangulation, Reverse-use dependency, Instability and Dispersion, all of which are potential predictor of proarrhythmias, ${ }^{b}$ Electrolyte concentration, and stimulation rate etc.

2006, Sugiyama 2008) can be divided into the following in vitro and in vivo models. Table $V$ shows the advantages and disadvantages of each model.

Rabbit or guinea pig Langendorff heart preparation with AV block (Screenit system): The model consists of a paced Langendorff-perfused female heart that records MAP, from which the following parameters are evaluated: $M A P D$, triangulation (indicative of changes of Phase 3 repolarization: $\mathrm{MAPD}_{30}$ to $M A P \mathrm{D}_{90}$ ), reverse-use dependency (indicating that the MAPD is more prolonged at slower heart rate), instability (indicative of beat to beat MAPD variability), and spatial dispersion (indicative of the difference between the MAPD of epicardium and endocardium).

Dog, rabbit, and feline left ventricular wedge preparation: The preparation is arterially perfused via its native coronary artery bed so that all ventricular myocardial layers are physiologically coupled, closely resembling in vivo models. Transmembrane action potentials can be recorded from these three layers (epicardium, mid cells and endocardium) simultaneously. The parameters examined are MAPD, triangulation, reverse-use dependency, instability, and transmural dispersion.

Rabbit methoxamine-sensitized anesthetized model (Carlsson model): $\alpha$-choloralose anesthetized rabbits treated with methoxamine ( $\alpha_{1}$ agonist) are used. The parameter examined is the direct index of proarrhythmia such as TdP-like VT and VF. Caution is needed when screening drugs with ancillary $\alpha$-adrenoceptor blocking properties because the effect may antagonize the proarrhythmic potential of drugs. QT or MAPD can be recorded but in particular the accurate measurement of QT interval is relatively difficult because $T$ wave may overlap the $P$ wave at fast heart rate or bigeminy is likely to develop during methoxamine infusion alone.

Dog or monkey with chronic atrio-ventricular (AV) block model: Anesthetized or conscious dogs and/or monkeys with chronic idioventricular bradycardia after complete AV block are utilized. This model expresses ventricular remodeling as a response to bradycardia-induced volume overload. The parameters examined are MAPD, triangu- 
lation, reverse-use dependency, instability, and spatial dispersion (in case of anesthetized state) as well as QT interval and the direct indexes of proarrhythmias such as TdP-like VT and VF.

\section{CONCLUSION}

The electrophysiological mechanisms that underlie the development of TdP are complex and influenced by wide variety factors that are not completely understood. Therefore, a negative result in our screen program would not completely rule out the QT liability and TdP risk of drug candidates in humans. Furthermore, it has been widely-recognized important to detect TdP due to $\mathrm{hERG}$ channel trafficking inhibition and VT and/or VF with QT shortening of drug candidates, both of which have not been addressed in the ICH S7B guideline. This development program, therefore, will need to be revised in line with latest-scientific knowledge in this field, and we adopt this development program, with insight into the advantages and limitations of each test, to distinguish drugs which prolong or shorten the QT interval but are not proarrhythmic from those that affect ECG morphology including QT interval and lead to fatal arrhythmia, not at the expense of discarding therapeutically innovative drugs.

\section{REFERENCES}

Belardinelli, L., Shryock, J. C., Wu, L. and Song, Y. (2005). Use of preclinical assays to predict risk of drug-induced torsades de pointes. Heart Rhythm. 2, S16-S22.

Camm, A. J. (2005). Clinical trial design to evaluate the effects of drugs on cardiac repolarization: current state of the art. Heart Rhythm. 2, S23-S29.

Carlsson, L. (2006). In vitro and in vivo models for testing arrhythmogenesis in drugs. J. Internal. Medicine. 259, 70-80.

Carlsson, L. (2008). The anaesthetised methoxamine-sensitised rabbit model of torsades de pointes Pharmacol. Ther. 119, $160-167$.

Cavero, I., Mestre, M., Guillon, J. M. and Crumb, W. (2003). Drugs that prolong QT interval as an unwanted effect: Assessing their likelihood of inducing hazardous cardiac dysrhythmias. Expert Opin. Pharmacother. 1, 947-973.

Dumotier, B. M. and Georgieva, A. V. (2007). Preclinical cardio-safety assessment of torsadogenic risk and alternative methods to animal experimentation: the inseparable twins. Cell Biol. Toxicol. 23, 293-302.

Furuhama, K. (2007). Toxicology assessment of fluoroquinolones: A case study. In Nonclinical Drug Safety Assessment: Practical Considerations for Successful Registration (Sietsema, W. K. and Schwen R., Eds.), pp 571-588. FDA news, Falls Church, USA.

Hashimoto, K. (2008). Torsades de pointes liability inter-model comparisons: The experience of the QT PRODACT initiative.
Pharmacol. Ther. 119, 195-198.

Hanson, L. A., Bass, A. S., Gintant, G., Mittelstadt, S., Rampe, D. and Thomas, K. (2006). ILSI-HESI cardiovascular safety subcommittee initiative: evaluation of three non-clinical models of QT prolongation. J. Pharmacol. Toxicol. Methods. 54, 116-129.

Hoffmann, P. and Warner, B. (2006). Are hERG channel inhibition and QT interval prolongation all there is in drug-induced torsadogenesis? A review of emerging trends. J. Pharmacol. Toxicol. Methods. 53, 87-105.

Liu, T., Brown, B. S., Wu, Y., Antzelevitch, C., Kowey, P. R. and Yan, G. X. (2006). Blinded validation of the isolated arterially perfused rabbit ventricular wedge in preclinical assessment of drug-induced proarrhythmias. Heart Rhythm. 3, 948-956.

Lu, H. R., Vlaminckx, E., Hermans, A. N., Rohrbacher, J., Van, Ammel. K., Towart. R., Pugsley, M. and Gallacher, D. J. (2008). Predicting drug-induced changes in QT interval and arrhythmias: QT-shortening drugs point to gaps in the ICHS7B Guidelines. Br. J. Pharmacol. 154, 1427-1438.

Milberg, P., Eckardt, L., Bruns, H. J., Biertz, J., Ramtin, S., Reinsch, N., Fleischer, D., Kirchhof, P., Fabritz, L., Breithardt, G. and Haverkamp, W. (2002). Divergent proarrhythmic potential of macrolide antibiotics despite similar QT prolongation: Fast Phase 3 repolarization prevents early after depolarizations and torsade de pointes $\mathrm{J}$. Pharmacol. Exper. Ther. 303, 218-225.

Oros, A., Beekman, J. D. and Vos, M. A. (2008). The canine model with chronic, complete atrio-ventricular block. Pharmacol Ther. 119, 168-178.

Pollard, C. E., Valentin, J. P. and Hammond. T. G. (2008). Strategies to reduce the risk of drug-induced QT interval prolongation: a pharmaceutical company perspective. Br. J. Pharmacol. 154, 1538-1543.

Redfern, W. S., Carlsson, L., Davis, A. S., Lynch, W. G. MacKenzie, I., Palethorpe, S., Siegl, P. K., Strang, I., Sullivan, A. T., Wallis, R., Camm, A. J. and Hammond, TG. (2003). Relationship between preclinical cardiac electrophysiology, clinical QT interval prolongation and torsade de pointes for a broad range of drugs: Evidence for a provisional safety margin in drug development. Cardiovasc. Res. 58, $32-45$.

Sugiyama, A. (2008) Sensitive and reliable proarrhythmia in vivo animal models for predicting drug-induced torsades de pointes in patients with remodelled hearts. Br. J. Pharmacol. 154, 1528-1537.

Tashibu, H., Miyazaki, H., Aoki, K., Akie, Y. and Yamamoto, K. (2005). QT PRODACT: in vivo QT assay in anesthetized dog for detecting the potential for QT interval prolongation by human pharmaceuticals. J. Pharmacol. Sci. 99, 473-486.

Thomsen M. B., Matz, J., Volders P. G. A. and Vos M. A. (2006). Assessing the proarrhythmic potentials of drugs: Current status of models and surrogate parameters of torsades de pointes arrhythmia. Pharmacol. Ther. 112, 150-170.

Toyoshima, S., Kanno, A., Kitayama, T., Sekiya, K., Nakai, K., Haruna, M., Mino, T., Miyazaki, H., Yano, K. and Yamamoto, K. (2005). QT PRODACT: in vivo QT assay in the conscious dog for assessing the potential for QT interval prolongation by human pharmaceuticals. J. Pharmacol. Sci. 99, 459-471.

Valentin, J. P., Hoffmann, P., De Clerck, F., Hammond, T. G. and Hondeghem, L. (2004). Review of the predictive value of the Langendorff heart model (Screenit system) in assessing 
the proarrhythmic potential of drugs. J. Pharmacol. Toxicol. Methods. 49, 171-181.

Vik, T., Pollard, C. and Sager, P. (2008). Early clinical development: Evaluation of drug-induced torsades de pointes risk. Pharmacol. Ther. 119, 210-214.

Webster, R., Leishman, D. and Walker, D. (2002). Toward a drug concentration effect relationship for QT prolongation and torsades de pointes. Curr. Opin. Drug Discov. Devel. 5 ,
116-126.

Yao, X., Anderson, D. L., Ross, S. A., Lang, D. G., Desai, B. Z., Cooper, D. C., Wheelan, P., Mclntyre, M. S., Bergquist, M. L., MacKenzie, K. I., Becherer, J. D. and Hashim, M. A. (2008). Predicting QT prolongation in humans during early drug development using hERG inhibition and an anaesthetized guinea-pig model. Br. J. Pharmacol. 154, 14461456. 